\title{
Childhood non-Hodgkin's lymphoma in Cipto Mangunkusumo Hospital, Jakarta: Outcome of treatment 2000-2005
}

\author{
Djajadiman Gatot, Teny Tjitrasari, Novie Amelia Chozie
}

\begin{abstract}
Background Childhood non-Hodgkin's lymphoma (NHL) is the third most common solid tumor in Cipto Mangunkusumo, Jakarta. Since 1992 there was no national report on the survival of children with $\mathrm{NHL}$. To continue our observation on the result of treatment of children with $\mathrm{NHL}$ in our institution, we briefly report the outcome therapy of children with NHL who were admitted to our hospital during 20002005.

Objective All patients who were diagnosed as non-Burkitt type NHL between January 2000-December 2005 were included in the study. Data collected retrospectively from the Oncology Registration of Hematology-Oncology Division, Department of Child Health, Cipto Mangunkusumo Hospital, including age, sex, primary site of tumor, histopathology type, staging, treatment response, and outcome.

Results A total of 24 patients were available. Male:female ratio was 1.8:1. The age range was from 9 months to 11 years (median 6 years). The histological type consisted of LL (3) and non-LL (11). Ten out of 14 patients were diagnosed as advanced stages (stages III and IV), while the rest were in stage II. Primary tumor site in LL type were the head and neck (1), mediastinum (1), and testis (1), while the non-LL type patients had more varied site. Overall survival of $\mathrm{NHL}$ was $78.6 \% \pm 4.7 \%$.

Conclusion The overall survival of childhood NHL patients treated with protocol in our institution is in the range of survival that had achieved in other centers worldwide, even with advanced stage of disease.[Paediatr Indones 2006;46:185-188].
\end{abstract}

Keywords: non-Hodgkin's lymphoma, childhood, outcome

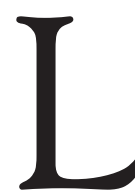

ymphomas (Hodgkin's and non Hodgkin's) constitute $10 \%-15 \%$ of all childhood cancers in the developed countries after acute leukemias and brain tumors. ${ }^{1,2}$ Non-Hodgkin lymphoma (NHL) is the third most common solid tumor during 2000-2005 in Cipto Mangunkusumo Hospital (CMH), Jakarta.

In CMH, during 1986-1991, there were 28 cases of non-Hodgkin's lymphoma treated with chemotherapy. About $71 \%$ (20 out of 28) came with late stage disease, consisted of 7 out of 28 patients stage III and 13 out of 28 patients stage IV, and none of these children were diagnosed as stage I. Of the 28 children who received full course of induction treatment, 15 achieved complete remission while the rest of them passed away during induction as the result of advanced disease with or without severe complications. ${ }^{3}$ Since then there is no further report on outcome of childhood NHL in our hospital.

The aim of this study is to continue our observation and to report the current outcome therapy of childhood NHL who were treated in our hospital.

\section{Methods}

All patients who were diagnosed as non-Burkitt type NHL during January 2000-December 2005 were

From the Department of Child Health, Medical School, University of Indonesia, Jakarta, Indonesia.

Reprint requests to: Djajadiman Gatot, MD, Hematology-Oncology Division, Department of Child Health, Medical School, University of Indonesia, Cipto Mangunkusumo Hospital, Jl. Salemba 6, Jakarta, Indonesia. Tel.62-21-31091170. Email: djagat_ps@yahoo.com 
included in the study. The Burkitt type NHL patients were excluded because the standard protocol was different. Data collected retrospectively from the Oncology Registration of Hematology-Oncology Division, Department of Child Health, Cipto Mangunkusumo Hospital, including age, sex, primary site of tumor, histopathology type, staging, treatment response, and outcome.

Diagnosis was based on histopathology category according to the World Health Organization classification. ${ }^{4}$ The cell surface markers were not examined because the test was expensive. Investigations to determine the stage of the disease included physical examination, complete blood cell count, imaging studies: X-ray, ultrasonography and CT-scan, and bone marrow aspiration were carried out. The staging system referred to that proposed at the St. Jude Children's Research Hospital, which takes into account primary site as well as extent of the disease. ${ }^{1}$

The standard protocol for childhood non Burkitt type NHL consists of:

- Induction. The induction regimen consists of vincristine (VCR) $1.5 \mathrm{mg} / \mathrm{m}^{2}$ weekly for 6 weeks, cyclophosphamide (CPA) $200 \mathrm{mg} / \mathrm{m}^{2}$ weekly for 6 weeks and prednisone $40 \mathrm{mg} / \mathrm{m}^{2} /$ day for 5 weeks followed by tapered off dose within 1 week. For stage III and IV diseases, adriamycin (ADR) 45 $\mathrm{mg} / \mathrm{m}^{2}$ twice weekly for 3 weeks were added.

- Maintenance. Oral metothrexate (MTX) (30 mg/ $\mathrm{m}^{2} /$ week), 6-mercaptopurine (6-MP) $\left(65 \mathrm{mg} / \mathrm{m}^{2} /\right.$ day), and CPA $\left(200 \mathrm{mg} / \mathrm{m}^{2} /\right.$ week) with VCR $\left(1.5 \mathrm{mg} / \mathrm{m}^{2} /\right.$ week $)$ and prednisone $\left(40 \mathrm{mg} / \mathrm{m}^{2} /\right.$ day for 2 weeks) every 3 months were planned to be given as maintenance treatment for at least 18 months.

- CNS prophylaxis. IT MTX (12 mg/m² weekly x 5) was used for CNS prophylaxis and was administered during induction phase.

- CNS disease treatment. Same as above but given twice weekly, until 3 consecutive normal spinal fluids. This was followed by cranial irradiation (1800 cgray), given when remission was achieved.

- Radiotherapy. No irradiation was done except for those with symptoms of airway obstruction.

Treatment response was evaluated after induction phase and at the end of chemotherapy based on clinical features, relevant laboratory, and imaging results.
The analysis is based on follow-up data as of July 2006. In the analysis of the outcome, the dependent variable was overall survival-the time from the initiation of therapy to death for any reason. All data were analyzed by SPSS version 13 and life tables for overall survival were constructed according to the method of Kaplan and Meier.

\section{Results}

A total of 14 patients were entered to study during January 2000-December 2005. Patient characteristics are shown in Table 1 . The male and female ratio was 1.8:1. The age range was from 9 months to 11 years (median 6 years).

Of 14 patients with NHL, 3 had lymphoblastic lymphoma (LL) and 11 had non-LL histological type. Ten patients came to our hospital in advanced stage (stage III and IV), while the rest came in stage II and none of them was diagnosed as stage I (Table $2)$. The 3 LL type patients presented with primary tumor in the head (1), mediastinum (1), and testis

Table 1. Characteristics of 14 patients

\begin{tabular}{lc}
\hline \multicolumn{1}{c}{ Characteristics } & Total \\
\hline Age & \\
$\leq 5$ & 5 \\
$>5$ & 9 \\
Gender & \\
Male & 9 \\
Female & 5 \\
Histology & \\
LL\# & 3 \\
Non LL* & 11 \\
St Jude staging & \\
I & 0 \\
II & 4 \\
III & 5 \\
IV & 5 \\
\hline
\end{tabular}

\# LL: lymphoblastic lymphoma

* Non LL: small non cleaved cell, diffuse large cell lymphomas and others.

Table 2. Primary site of tumor listed by histology of 14 PATIENTS

\begin{tabular}{lccc}
\hline Primary site of tumor & LL & Non LL & Total \\
\hline Head \& neck & & & \\
$\quad$ Eye & 0 & 2 & 3 \\
Lymph node & 1 & 6 & 7 \\
$\quad$ Parotid & 0 & 1 & 1 \\
Mediastinum & 1 & 1 & 2 \\
Testis & 1 & 0 & 1 \\
\hline
\end{tabular}


Table 3. Histology and St. Jude staging of 14 PATIENTS

\begin{tabular}{lccccc}
\hline & \multicolumn{5}{c}{ St. Jude Staging } \\
& I & II & III & IV & \\
\hline LL & 0 & 0 & 0 & 3 & 3 \\
LCL & 0 & 3 & 2 & 1 & 6 \\
SNC & 0 & 0 & 1 & 1 & 2 \\
Others & 0 & 1 & 2 & 0 & 3
\end{tabular}

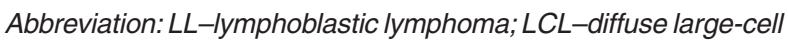
lymphoma; SNC-small non-cleaved cell lymphoma.

(1); while the non-LL type patients had more varied primary tumor site (Table 3 ).

The projected overall survival after diagnosis of Non Hodgkin's lymphoma was $78.6 \%$ (95\%CI 72.2; 85.0\%) (Figure 1).

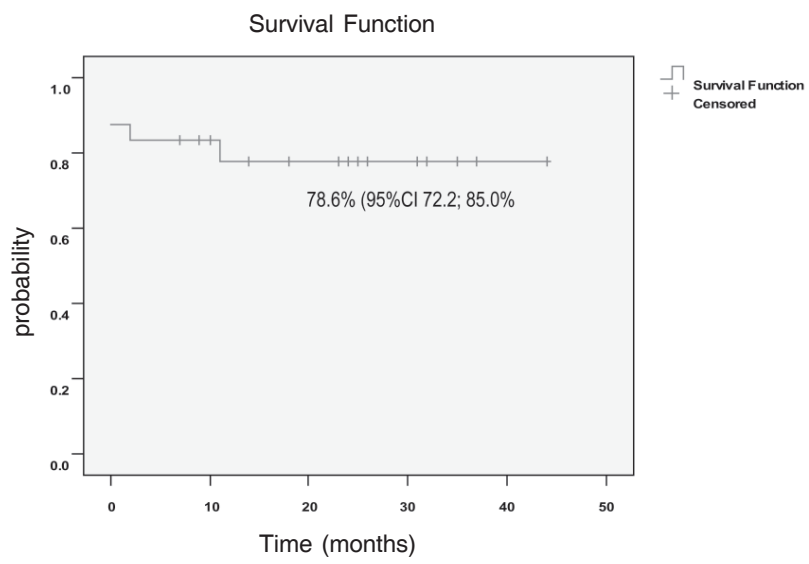

Figure 1. OVerall survival of 14 Patients

\section{Discussion}

Our study showed that patient aged more than 5 years was almost twice as high as patient aged under 5 years. Unlike Hodgkin's disease, which has a bimodal incidence curve, the incidence of non-Hodgkin's lymphoma increases steadily with age throughout life. The incidence of non-Hodgkin's lymphoma is much lower in children compared with adults. ${ }^{1,2}$ The ratio of male and female patients in our study was 1.8:1. These results are consistent with other reported studies. ${ }^{1,5-7}$

Clinical presentations of NHL in children are varied and depend on the histological subtype, the extent (or stage) of the disease, and the primary site of tumor. ${ }^{1}$ LL typically presents as a mediastinal or head and neck mass. ${ }^{8}$ Our study showed 3 patients with LL had different site of primary tumor, 1 in mediastinum, 1 in head and neck, and 1 in testis. Primary NHL of the testicle is rare. ${ }^{1}$ Lagrange et al ${ }^{9}$ reported 84 cases of primary NHL with median age 67 years (range $17-85$ years), about $10.7 \%$ has lymphoblastic histological type. Data of similar case in children is unknown. The non-LL (small noncleaved cell and large cell) type showed varied primary site of tumor in head and neck region and mediastinum. None of our patients had primary tumor in abdomen.

The prognosis for children with NHL has markedly improved during the past 20 years. In early 1970s, the survival rate was less than $30 \% .{ }^{10}$ Recently, the survival rate of $65-75 \%$ for advanced LL and $75-90 \%$ for advanced Burkitt's and other B-lineage lymphomas are reported. Even patients with advanced disease currently exhibit $65-90 \%$ projected event free survival rate. ${ }^{11-12}$ Our study showed the overall survival of childhood NHL was $78.6 \%+4.7 \%$ (SE). We divided the histology type in data presentation and analysis into LL and non-LL because the two forms has different prognosis. The LL type has poor prognosis compare to non-LL type. ${ }^{1,11}$ Survival rate for each histological type and event-free survival rate could not be constructed in our study because the number of patients is small.

Analysis of the result of treatment according to the histological subtypes of non-Hodgkin's lymphoma revealed important differences. Our study showed that all patients with LL came to our hospital in the late stage (stage IV). LL has poorer prognosis than other histological subtypes. ${ }^{1,8}$ The event-free survival of the patients with LL; a histological subtype that accounts for approximately $15 \%$ of NHL in children and young adults-was inferior to that of the patients with other subtypes of lymphomas. ${ }^{5}$ Link et al ${ }^{5}$ found that only $63 \%$ of the patients with early-stage LL were projected to survive without a recurrence of disease for five years, as compared with a projected $89 \%$ of patients with small non-cleaved-cell lymphomas and $88 \%$ of those with large-cell lymphomas $(\mathrm{P}<0.001)$. Advani et al ${ }^{6}$ showed that event free survival (EFS) was $48 \%$ for LL and 68\% for non-LL; while for all patients was $58 \%$.

NHL is best regarded as a systemic disease due to the unique anatomy of the lymphoid system and physiology of lymphoid cells, which tend to migrate whether they are normal or malignant. Treatment protocols in 
childhood NHL are designed to maximize benefit and minimize toxicity. At the end, the best indicator of the ultimate outcome of therapy is the response to treatment itself, so that a measure of the response to therapy in the early treatment cycles can be used.

In conclusion, the overall survival of childhood NHL patients treated with protocol in our institution is in the range of survival that had achieved in other centers worldwide, even with advanced stage of diseases. However, the number of patients in our study is too small. We need to continue this study with larger number of patients and longer time of observation to analyze the event free survival rate.

\section{Acknowledgement}

We thank Ferry Damardjati, MD; Rosary, MD; and Yuni for their assistance in statistical analysis.

\section{References}

1. Magrath IT. Malignant non-Hodgkin's lymphomas in children. In: Pizzo PA, Poplack DG, editors. Principles and practice of pediatric oncology. Lippincott Williams \& Wilkins; Philadelphia: 2002, p. 661-705.

2. Johnston JM. Non-Hodgkin lymphoma. Cited from www.emedicine.com.

3. Djajadiman Gatot, Idham Amir. Childhood nonHodgkin's lymphoma in Cipto Mangunkusumo Hospital Jakarta: The obstacles in treatment and follow-up. Indonesian Journal of Pathology;1992:4042.

4. Harris NL, Jaffe ES, Diebold J, Flandrin G, MullerHermelink HK, Vardiman J, et al. World Health Organization classification of neoplastic diseases of the hematopoietic and lymphoid tissues: Report of the clini- cal advisory committee meeting-Airlie House, Virginia, November 1997. J Clin Oncol 1999;17:3835-49.

5. Link MP, Shuster JJ, Donaldson SS, Berard CW, Murphy SB. Treatment of children and young adults with early-stage non-Hodgkin's lymphoma. N Engl J Med 1997;337:1259-66.

6. Advani S, Pai S, Adde M, Vaidya S, Vats T, Naresh K, et al. Preliminary report of an intensified, short duration chemotherapy protocol for the treatment of pediatric non-Hodgkin's lymphoma in India. Ann Oncol 1997;8:893-7.

7. Kobrinsky NL, Sposto R, Shah NR, Anderson JR, DeLaat C,Morse M, et al. Outcomes of treatment of children and adolescents with recurrent non-Hodgkin's lymphoma and Hodgkin's disease with dexamethasone, etoposide, cisplatin, cytarabine and L-Asparaginase, maintenance chemotherapy and transplantation: Children's Cancer Group Study CCG-5912. J Clin Oncol 2001;19:2390-96.

8. Sandlund JT, Downing JR, Crist WM. Non-Hodgkin Lymphoma in childhood. NEJM 1996;334:1238-48.

9. Lagrange JL, Ramaioli A, Theodore C, Terrier-Lacombe MJ, Beckendorf V, Biron P, et al. Non-Hodgkin's lymphoma of the testis: A retrospective study of 84 patients treated in the French anticancer centres. Ann Oncol 2001;12:1313-19.

10. Murphy SB, Frizzera G, Evans AE. A Study of childhood non-Hodgkin lymphoma. Cancer 1975;36:212131.

11. Weinstein HJ, Cassady JR, Levey R. Long-term results of the APO protocol (vincristine, doxorubicin/adriamycin and prednisone) for treatment of mediastinal lymphoblastic lymphoma. J Clin Oncol 1983;1:537-41.

12. Dahl GV, Rivera G, Pui CH, Mirro J, Ochs J, Kalwinsky DK, et al. A novel treatment of childhood lymphoblastic non-Hodgkin's lymphoma: Early and intermittent use of teniposide plus cytarabine. Blood 1985:66;1110-14. 